\title{
Heuristic model for the energy spectrum of phase turbulence
}

\author{
J. T. N. Medeiros and A. S. da Rosa Simões \\ Instituto de Física, Universidade Federal do Rio Grande do Sul, 91501-970 Porto Alegre, RS, Brazil
}

(Received 5 April 2001; published 30 October 2001)

\begin{abstract}
We present a heuristic model for the energy spectrum of the one-dimensional phase turbulence in the steady state of the Kuramoto-Sivashinsky equation. Our model contains an energy transfer mechanism from low- to high-wave-vector modes. The energy transfer is written as the sum of local and nonlocal interactions. Our analytical results show good agreement with numerical simulations, particularly for the hump in the energy spectrum, which is mainly due to the local interactions.
\end{abstract}

DOI: 10.1103/PhysRevE.64.057301 PACS number(s): 47.27.Eq, 05.45. $-\mathrm{a}, 47.52 .+\mathrm{j}, 47.54 .+\mathrm{r}$

A satisfactory understanding of spatially extended systems, although fascinating, is a difficult task. The evolution of these systems is generally described by nonlinear partial differential equations where analytical results are rather scarce [1]. Phase turbulence is the irregular behavior of an extended system described by the paradigmatic KuramotoSivashinsky equation (KSE), one of the simplest partial differential equations exhibiting chaotic behavior [2]. It appears in a variety of physical systems driven away from equilibrium such as reaction-diffusion chemical systems [3] or flame front propagation [4]. In one dimension it reads

$$
\varphi_{t}+\frac{1}{2} \varphi_{x}^{2}+\nu \varphi_{x x}+\mu \varphi_{x x x x}=0
$$

with $\nu, \mu>0$. Defining $u=\varphi_{x}$ as the velocity field, one gets the alternative equation

$$
u_{t}+u u_{x}+\nu u_{x x}+\mu u_{x x x x}=0 .
$$

This equation can also be written in the standard scaled form $u_{t}+u u_{x}+u_{x x}+u_{x x x x}=0$. The periodic boundary conditions normally used are $u(x+L)=u(x), u_{x}(x+L)=u_{x}(x)$, etc. $L$ is the length of the system. In the thermodynamic limit ( $L$ $\rightarrow \infty$ ) there is no free control parameter. The unstable growth of fluctuations given by the term $\nu u_{x x}$ acts as an energy source in the large-wavelength region. In the shortwavelength region the fluctuations are attenuated by the term $\mu u_{x x x x}$ which acts as a stabilizing energy sink. We can say that $\nu$ and $\mu$ play the roles of an "antiviscosity" and a "hyperviscosity," respectively.

Several papers have addressed the study of the longwavelength behavior - the hydrodynamic limit — of the KSE in one dimension. It was conjectured by Yakhot [5], using a perturbative renormalization group approach, that the statistical behavior of the KSE, written as in Eq. (2), is equivalent to the stochastic Burgers equation [6] $u_{t}+u u_{x}-\tilde{\nu} u_{x x}+\eta_{u}$ $=0$, where $\tilde{\nu}>0$ is a renormalized viscosity and $\eta_{u}$ is a Gaussian white noise forcing. Alternatively, the KSE written as in the Eq. (1), is equivalent to the Kardar-Parisi-Zhang (KPZ) equation [7] $\varphi_{t}+\varphi_{x}^{2} / 2-\tilde{\nu} \varphi_{x x}+\eta_{\varphi}=0$. The numerical work of Sneppen et al. [8] strongly supports Yakhot's conjecture. An analytical demonstration of the connection between KS and KPZ equations was given by Chow and Hwa [9] by explicitly coarse-graining the KSE. Generically, the system forms cells of a preferred size. These cells are locally compressed or stretched giving rise to cell creation or annihilation. This mechanism provides a positive renormalized viscosity $\tilde{\nu}$ and the cell interactions are sufficiently uncorrelated to give rise to the random forcing $\eta$.

One of the quantities of primary interest when the KSE is numerically integrated is the mean energy of the $k$ mode, $E(k)$, which is the Fourier transform of the two-point correlation function of $u(x, t)$. Numerical simulation results for the energy spectrum $E(k)$ have shown three main characteristics: (i) a flat region for low wave number $k$, (ii) a hump near $k=1$, and (iii) a strongly decaying region for high $k$ [10-14]. Despite the paradigmatic relevance of this model there are only a few analytical results for $E(k)$, just two as far as we know. Pomeau et al. [10] considered a local energy flux as the product of $E(k)$ with an $a d$ hoc $k^{4}$ factor in order to take into account the assumed $k^{-4}$ power law in the observed spectrum. They obtained a constant energy flux by ignoring energy injection. In particular, the flat region of the spectrum is absent. Toh [11] obtained $E(k)$ by a statistical model in which pulses with imposed rigid shapes are created and annihilated. The rigid shapes were calculated by numerically solving the static KSE $\left(u_{t}=0\right)$.

In this work we calculate the energy spectrum analytically, using a heuristic model for the energy transfer among modes, resulting in very good agreement with the numerical simulations. First of all, we derive a basic dynamic equation for equal time correlation functions of the KSE. Using $u$ $=u(x, t)$ and $u^{\prime}=u\left(x^{\prime}, t\right)=u(x+r, t)$, we easily find

$$
\frac{\partial\left(u^{\prime} u\right)}{\partial t}+\frac{1}{2} \frac{\partial\left(u^{2} u^{\prime}-u^{\prime 2} u\right)}{\partial r}+2\left[\nu \frac{\partial^{2}}{\partial r^{2}}+\mu \frac{\partial^{4}}{\partial r^{4}}\right] u^{\prime} u=0 .
$$

Taking the spatial average of the above equation we obtain

$$
\frac{\partial C_{2}(r, t)}{\partial t}+\frac{\partial C_{3}(r, t)}{\partial r}+2\left[\nu \frac{\partial^{2}}{\partial r^{2}}+\mu \frac{\partial^{4}}{\partial r^{4}}\right] C_{2}=0
$$

where $C_{2}(r, t)=\left\langle u^{\prime} u\right\rangle$ and $C_{3}(r, t)=\frac{1}{2}\left\langle\left(u^{2} u^{\prime}-u^{\prime} u\right)\right\rangle$ are, respectively, the two- and three-point correlation functions. Equation (4) is similar to the von Kármán-Howarth equation for fluid turbulence [15]. The energy spectrum is defined by 
$E(k)=U^{*}(k) U(k)$, where $U(k)$ are the Fourier amplitudes of the velocity field $u(x)=\int d k e^{i k x} U(k)$. The spectral form of Eq. (4) reads

$$
\partial E(k, t) / \partial t=2 \nu k^{2} E+T(k)-2 \mu k^{4} E,
$$

which is fundamental in this work. In the above equation the term $2 \nu k^{2} E \equiv I$ is called the energy injection and $2 \mu k^{4} E$ $\equiv D$ the energy dissipation. The term $T(k)$ arises from the nonlinear term and redistributes the energy among the individual spectral modes. It is given by

$$
\begin{gathered}
T(k)=\int S(k ; p, q) d q d p, \\
S(k ; p, q)=-\operatorname{Im}[k U(k) U(p) U(q) \delta(k+p+q)] .
\end{gathered}
$$

In the steady state $\partial E(k, t) / \partial t=0$ and we can easily see that $T$ is subject to the constraint $\int_{0}^{\infty} d k T(k)=0$. This important integral condition shows us that no energy is generated or lost by $T$, but it is redistributed among the modes. In other words, the transfer mechanism cannot directly affect the energy injection $I$ or the dissipation $D$. There is a net energy flow from small to high $k$ and it can be stated as $I+T=D$. With the previous constraint we can express $T$ as

$$
T(k)=-\frac{\partial \Pi(k)}{\partial k}
$$

where $\Pi(k)$ is an energy flux due to the nonlinear term. The steady state for the energy spectrum can be rewritten in the integral form

$$
\Pi(k)=-\int_{0}^{k} T(p) d p=\int_{0}^{k}[I(p)-D(p)] d p .
$$

All previous expressions are exact and to solve them one needs some approximation to disconnect the three- from the two-point correlation functions $C_{3}$ and $C_{2}$ in Eq. (4). Expressing $\Pi$ as a function of $k$ and $E(k)$, Eq. (5) is immediately closed. Our first assumption for closing Eq. (5) is to write $\Pi$ as the sum of local $\Pi_{L}$ and nonlocal $\Pi_{N L}$ contributions:

$$
\Pi=\Pi_{L}+\Pi_{N L}
$$

For $\Pi_{L}$ we admit the energy transfer as coming from the injection $I$ and dissipation $D$ at the same $k$ value,

$$
\Pi_{L}=S(k) .
$$

For $\Pi_{N L}$ we assume that the energy flux at a particular $k$ depends on all values of $k$,

$$
\Pi_{N L}(k)=\int_{0}^{k} d p \int_{k}^{\infty} d q S(p, q),
$$

where $S(p, q) d p d q$ can be interpreted as the specific rate of transferred energy from modes in the range $(p, p+d p)$ to modes in the range $(q, q+d q)$. Contrasting with the ap- proach of fluid turbulence models in the inertial subrange, we have $S(q, p) \neq-S(p, q)$, which means that we have a net energy transport.

We propose a heuristic model for $\Pi$ based on dimensional analysis in the Kolmogorov way [15]. From the definition of the velocity field $u$ and parameters $\nu$ and $\mu$ in the KSE, we have the following dimensions: $[u]=\mathrm{LT}^{-1},[\nu]=\mathrm{L}^{2} \mathrm{~T}^{-1}$, $[\mu]=\mathrm{L}^{4} \mathrm{~T}^{-1}$. Equation (5) gives $[\partial E(k) / \partial t]=[T(k)]=[I]$ $=[D]=\mathrm{L}^{3} \mathrm{~T}^{-3}$. We assume that the $T$ dependence on $I$ and $D$ for the local case is on the same basis so we take it as the geometric mean of $I$ and $D: T_{L} \propto \sqrt{I D} \propto\left[\left(\nu k^{2} E\right)\left(\mu k^{4} E\right)\right]^{1 / 2}$ $=(\nu \mu)^{1 / 2} k^{3} E$ and $S(k) \propto(\nu \mu)^{1 / 2} k^{4} E(k)$, a kind of Pao model [15]. For the nonlocal case we will assume $S(q, p)$ $\propto p^{m} p E(p)^{n} p q^{m} q E(q)^{n} q$, similar to the von Kármán model [15]. From dimensional considerations $n_{p}+n_{q}=1$ and $m_{p}$ $+m_{q}=2$. It is physically reasonable to put $n_{p}=n_{q}=1 / 2$ since we assume that $I$ and $D$ are on the same footing. For simplicity we also assume only positive integers for $m_{p}$ and $m_{q}$ and we can easily see that the only possibilities for a net flux from low to high $k$ are $m_{p}=2$ and $m_{q}=0$. Assuming $\mu=\nu=1$ from now on, which means a rescaling of the KSE, we finally obtain

$$
\begin{gathered}
S(p, q)=\gamma p^{2} E^{1 / 2}(p) E^{1 / 2}(q), \\
S(k)=\lambda k^{4} E(k) .
\end{gathered}
$$

The model parameters $\gamma$ and $\lambda$ are dimensionless.

We are now able to obtain a differential equation for the spectral function. Writing the integral Eq. (8) with the model expressions for $\Pi_{L}$ and $\Pi_{N L}$ we get

$$
\begin{aligned}
& -\gamma\left\{\int_{0}^{k} e(p) p^{2} d p \int_{k}^{\infty} e(q) d q\right\}-\lambda\left\{k^{4} e^{2}(k)\right\}=2 \int_{0}^{k} d p\left(p^{4}\right. \\
& \left.-p^{2}\right) e^{2}(p)
\end{aligned}
$$

with the definition $\sqrt{E(k)}=e(k)$. Deriving this equation three times with respect to $k$, rearranging the terms, and defining $e(k)=f(k) / k^{2}$, we finally obtain the following homogeneous differential equation:

$$
\lambda k^{3} f^{\prime \prime \prime}+\left(k^{3}+3 \lambda k^{2}-k\right) f^{\prime \prime}+\left(3 k^{2}-\gamma k+1\right) f^{\prime}=0 .
$$

This is a very interesting equation and despite its linear form we were not able to find exact solutions, except the trivial $f=$ const. Nevertheless, we get particular solutions in two cases.

Local case. $\gamma=0$, corresponding to a local energy transfer. The energy spectrum is $E_{L} \propto k^{-4} e^{-(k+1 / k) / \lambda}$.

Nonlocal case. $\lambda=0$, corresponding to a nonlocal energy transfer. An interesting solution in this case, without poles and zeros in the energy spectrum, corresponds to $\gamma=3 \mathrm{im}-$ plying $E_{N L} \propto(1+k)^{-8}$.

In order to obtain the well established result from numerical simulations that show a flat region in the energy spectrum for $k \rightarrow 0$, the condition $\gamma / \lambda=3$ must be fulfilled in Eq. (14). This relevant condition links the local and nonlocal parameters $\lambda$ and $\gamma$. It implies a null flux of energy $(\Pi=0)$ in the absence of dissipation $(D=0)$. In the limit of $k \rightarrow \infty$ the re- 
sult emerging from Eq. (14) gives $E \propto k^{-8}$, corresponding also to a null flux, now in the absence of injection $(I=0)$.

We solve the differential equation analytically in the lowand high- $k$ limits and obtain the following nontrivial solutions:

$$
\begin{gathered}
e(k \rightarrow 0)=B_{0}\left[1+b_{0} g_{0}(k)\right], \\
g_{0}=e^{-3 / k}\left[3+3 k+k^{2}\right] / k^{2} ;
\end{gathered}
$$

and

$$
\begin{gathered}
e(k \rightarrow \infty)=B_{\infty}\left[1 / k^{4}+b_{\infty} g_{\infty}(k)\right], \\
g_{\infty}=e^{-3 k}\left[1+3 / k+3 / k^{2}\right] / k^{2} .
\end{gathered}
$$

The above four parameters $B_{0}, b_{0}, B_{\infty}$, and $b_{\infty}$ are the integration constants written in a convenient way.

The complete energy spectrum is obtained by matching the two asymptotic solutions above with a third one, which is obtained as a series solution in the interval $\left[k_{l}, k_{h}\right]$ around the regular point $k=1$. This point separates the region $k$ $<1$ where $I$ dominates from the region $k>1$ where $D$ dominates. We rewrite Eq. (14) replacing the variable $k$ by $z=1$ $-k$ and we find the series solutions $h(z)$ near $z=0$. The three solutions are the trivial $h_{0}=$ const and the series solution $h_{1} \quad\left(h_{2}\right)$ beginning with a $z\left(z^{2}\right)$ term. Admitting that the factor $F=\exp [-(k+1 / k) / \lambda]$ from the local solution will survive near $k=1$ in the global solution, we find $F \simeq a+b z^{2}$ $+\cdots$ after expanding it around $z=0$. To fulfill the previous condition we are restricted to $h_{0}$ and $h_{2}$, resulting in the following solution:

$$
\begin{gathered}
e(k \approx 1)=B_{1}\left[1 / k^{2}+b_{1} g_{1}\right], \\
g_{1}(k)=h_{2}(1-k) / k^{2} .
\end{gathered}
$$

$B_{1}$ and $b_{1}$ are integration constants. We have to determine the six integration constants in Eqs. (15)-(17). Nevertheless, their number is reduced to only two by matching the solutions and their first derivatives at the matching points $k_{l}$ and $k_{h}$.

We have normalized the numerical results [10-14] to $E_{\text {max }}(k)=1$ and we observe that they are almost collapsed into a single curve with $E(0) \cong 0.0892$. We choose one of these curves to represent all the numerical results, the curve $Z$ in Fig. 1, which was obtained by Zalesky [12]. Using the numerical values for $E_{\max }(k)$ and $E(0)$ together with the matching points $k_{l}=0.5$ and $k_{h}=1.5$, eventually we obtain the complete energy spectra for different values of the local parameter $\lambda$. The nonlocal parameter $\gamma$ is linked to the local one by $\gamma / \lambda=3$.

Our results show an overall agreement with the numerical results and they are summarized by the curves $A, B$, and $L$ in

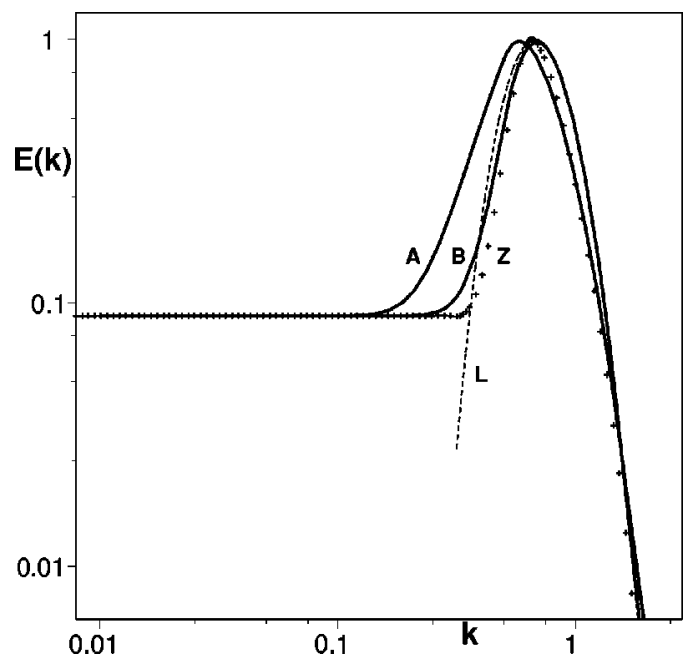

FIG. 1. Energy spectra for the Kuramoto-Sivashinsky equation: $A$ and $B$ are the results of our model with $\lambda=1 / 3$ and $1 / 6$, respectively. $L$ is our local model approximation. $Z$ are the numerical results of Zalesky [12].

Fig. 1. The curve $A$, corresponding to $\lambda=1 / 3$, shows a particular good fitting at the right side of the hump. This agreement is important since some authors $[1,10]$ admit the existence of an inertial subrange in this region. However, the maximum of the hump is shifted to left. The curve $B$, corresponding to $\lambda=1 / 6$, shows a better agreement with the hump, in particular near the maximum. All curves show the strong characteristic decay in the dissipative region of the spectrum. The curve $L$ represents only the local contribution for the energy flux and a good fitting is obtained with $\lambda$ $=1 / 5$. As we can see, the local contribution gives an adequate description of the spectrum in the irregular region. It may be seen as a manifestation of the division of thick cells and the coalescence of thin cells as in the Chow-Hwa model [9]. This mechanism, involving only local change of wave numbers and providing a band of states with nearby wave numbers, seems to be a convincing physical interpretation for our local model.

In conclusion, we have presented a heuristic model for the KSE energy spectrum based on dimensional analysis in the Kolmogorov way. The main characteristic of our model is that it contains a natural mechanism of energy transfer from low- to high- $k$ modes. The energy flow is given as the sum of local and nonlocal interactions. The analytical results provided by our model show overall excellent agreement with the numerical calculations. In particular, the plateau for low $k$, the hump at $k \approx 1$, and the decay for high $k$ are clearly observed. Our model does not have a strong dependence on the free parameter $\lambda$; however, the local part of the energy flux accounts for the strong hump in the spectrum.
[1] M.C. Cross and P.C. Hohenberg, Rev. Mod. Phys. 65, 851 (1993).

[2] H. Chaté and P. Manneville, in Turbulence: A Tentative Dic- tionary, edited by P. Tabeling and O. Cardoso (Plenum Press, New York, 1995).

[3] Y. Kuramoto and T. Tsusuki, Prog. Theor. Phys. 52, 1399 
(1974); Prog. Theor. Phys. Suppl. 64, 346 (1978).

[4] G.I. Sivashinsky, Acta Astron. 4, 1177 (1977); 6, 560 (1979).

[5] V. Yakhot, Phys. Rev. A 24, 642 (1981).

[6] J. M. Burgers, The Nonlinear Diffusion Equation (Riedel, Boston, 1974).

[7] M. Kardar, G. Parisi, and Y.C. Zhang, Phys. Rev. Lett. 56, 889 (1986).

[8] K. Sneppen, J. Krug, M.H. Jensen, C. Jayaprakash, and T. Bohr, Phys. Rev. A 46, R7351 (1992).

[9] C.C. Chow and T. Hwa, Physica D 84, 494 (1995).
[10] Y. Pomeau, A. Pumir, and P. Pelce, J. Stat. Phys. 37, 39 (1984).

[11] S. Toh, J. Phys. Soc. Jpn. 56, 949 (1987).

[12] S. Zalesky, Physica D 34, 427 (1989).

[13] M. Rost and J. Krug, Physica D 88, 1 (1995).

[14] H.D. Dankovics, P. Holmes, G. Berkoos, and J. Elesgaray, Physica D 90, 387 (1996).

[15] A. S. Monin and A.M. Yaglom, Statistical Fluid Mechanics (MIT Press, Cambridge, 1975), Vol. 2. 